\title{
Vorwort der Autoren
}

Der mathematischen Beschreibung verfahrenstechnischer und artverwandter Objekte kommt seit der Einführung von Prozeßrechnern zur Automatisierung von Produktionsprozessen und der Durchführung von Prozeßanalysen zur Intensivierung entscheidende Bedeutung zu. Im Prinzip stellt die mathematische Beschreibung des Objekts überhaupt erst die Grundlage zur Schaffung eines automatisierten Steuerungssystems und zur Ableitung wissenschaftlich begründeter Maßnahmen zur Prozeßintensivierung dar. Aus dieser Tatsache erklärt sich auch das große Interesse, das von Spezialisten der verschiedenartigsten Industriezweige der Schaffung mathematischer Modelle entgegengebracht wird.

Bekanntlich existieren die verschiedensten Methoden zur Modellbildung, d. h. zur Gewinnung einer mathematischen Beschreibung. Die Autoren gehen auf statistische Methoden ein, die auf der Auswertung von Daten basieren, die im wesentlichen bei passiven Versuchen, $d . h$. bei normaler Fahrweise des Objekts, gewonnen wurden. Nach Meinung der Autoren ist eine solche Methodik bei einer großen Anzahl von Fällen anderen Vorgehensweisen vorzuziehen. Das Ergebnis stellt dann eine mathematische Beschreibung des in Betrieb befindlichen Objekts dar.

Speziell wird auf Besonderheiten bei der Anwendung traditioneller Methoden der mathematischen Statistik unter industriellen Bedingungen eingegangen.

Das Buch wendet sich an einen breiten Kreis von Spezialisten, die auf dem Gebiet der Prozeßanalyse, Rationalisierung und der Automatisierung von Produktionsprozessen tätig sind. Dabei werden Kenntnisse über die Grundbegriffe der mathematischen Statistik vorausgesetzt.

Der mathematische Anhang soll dem Leser helfen, einige Wissenslücken aus der linearen Algebra und der Statistik von Zufallsgrößen und Zufallsprozessen zu schließen.

Die Autoren danken Prof. Dr.-Ing. G. K. KRUG und Dr.-Ing. G. F. FraARETOW für ihre wertvollen Hinweise und Ratschläge bei der Fertigstellung des Buches.

Für die Kapitel 1., 2., 5. zeichnet W. P. BorodJuK als Autor verantwortlich, während die Kapitel 3., 4., 6. von E. K. LEZKIJ niedergeschrieben wurden.

Alle Wünsche und kritischen Hinweise werden von den Autoren dankbar entgegengenommen.

Die Autoren 